\title{
ANISOTROPY OF THE DIAMAGNETIC SUSCEPTIBILITY IN 3,5-DIBROMO-p-AMINO- AND 3,5-DIBROMO-p-HYDROXYBENZOIC ACIDS
}

\author{
M.M. ABDel-Kader \\ Physics Department, Faculty of Science, Cairo University, Giza, Egypt \\ (Received January 28, 1992; revised version May 5, 1992)
}

\begin{abstract}
The mean molar susceptibility $\left(\chi_{M}^{\prime}\right)$ for the two compounds 3,5-dibromo-p-aminobenzoic acid, $\mathrm{Br}_{2} \mathrm{C}_{6} \mathrm{II}_{2}\left(p-\mathrm{NI}_{2}\right) \mathrm{COOH}$, and 3,5-dibromo-p-hydroxybenzoic acid, $\mathrm{Br}_{2} \mathrm{C}_{6} \mathrm{II}_{2}$ ( $p$-OII)COOII, has been determined. The principal molecular susceptibilities $K^{\|}$and $K^{\perp}$ have been evaluated. The magnetic anisotropy of each of the two compounds was obtained. From the atomic coordinates, as given by the $\mathrm{X}$-ray structure analysis of $\mathrm{Br}_{2} \mathrm{C}_{6} \mathrm{II}_{2}\left(p-\mathrm{NH}_{2}\right) \mathrm{COOII}$, the crystal susceptibilities $\left(\chi_{a}, \chi_{b}\right.$, and $\left.\chi_{c}\right)$ lia ve been calculated. The magnetic properties are interpreted in the light of the crystal structure.
\end{abstract}

PACS numbers: $35.20 . \mathrm{My}$

\section{Introduction}

The strong anisotropy of the diamagnetic susceptibility of benzenoid compounds is usually explained in terms of ring current [1-9]. An interesting discussion with the use of ring current concept has been given by Gomes [10].

In general, the principal molecular susceptibilities $\left(K^{\perp}\right.$ and $\left.K^{\|}\right)$can be cvaluated from the crystal susceptibilities $\left(\chi_{1}, \chi_{2}\right.$, and $\left.\chi_{3}\right)$ combined with the orientation of the molecule in the crystal. The details of this method was given elsewhere [9]. On the other hand, the diamagnetic anisotropy $\Delta K$ of the molecule can be calculated from the measured value of the molar susceptibility $\chi_{\mathrm{M}}$ according to the relation [11]:

$$
\Delta K=3\left(\chi_{\mathrm{M}}^{\mathrm{obs}}-K^{\|}\right),
$$

where $K^{\|}$is the in-plane susceptibility whose value can be determined from the sum of the atomic susceptibilities of the molecule as discussed by Hoarau [11] or from the local values of in-plane susceptibilities $\chi^{\| l}(\mathrm{Av})$ as worked out by Mason [12], Amos and Roberts [6], and by Long and Memory [13]. In our recent paper [1.4] these two different methods that are used for the calculation of $K^{\prime l}$ have been outlined. 
In this paper the molar magnetic susceptibility $\chi_{\mathrm{M}}^{\mathrm{obs}}$ as well as the anisotropy of the diamagnetic susceptibility of 3,5-dibromo-p-aminobenzoic acid and 3,5-dibromo- $p$-hydroxybenzoic acid have been investigated. To the best of our knowledge, the magnetic susceptibility of these two compounds has never been measured. Ilowever, the method described here was successfully used by the author for studying the magnetic properties of similar materials $[9,14,16]$.

\section{Experimental}

The two compounds studied in this paper are:

(a) 3,5-dibromo-p-aminobenzoic acid, $\mathrm{Br}_{2} \mathrm{C}_{6} \mathrm{II}_{2}\left(p-\mathrm{NH}_{2}\right) \mathrm{COOII}$,

(b) 3,5-dibromo-p-hydroxybenzoic acid, $\mathrm{Br}_{2} \mathrm{C}_{6} \mathrm{H}_{2}$ (p-OII)COOII.

The motivation of such choice is that the crystal structure of the first compound is known [15]. The hydrogen bonding system is therefore clear. The second compound is more or less similar to the first one.

Recrystallization from saturated solution of a mixture of equal amounts of ethyl alcohol and acetone has been carried out. The observed molar magnetic susceptibility $\chi_{M}^{\mathrm{obs}}$ of each compound has been accurately measured by means of Gouy method. The description of this method was given elsewhere [9].

\section{Results and discussion}

The observed and calculated values of the molar susceptibilites $\chi_{\mathrm{M}}^{\mathrm{obs}}$ and $\chi_{\mathrm{M}}^{\text {calc }}$ for the two compounds are shown in Table I. The method used for the calculation of $\chi_{\mathrm{M}}^{\text {calc }}$ was given elsewhere $[14,16]$. The diamagnetic susceptibilities used here for such calculations are

$$
\begin{aligned}
& \chi_{\text {benzene ring }}=-55.20[17], \quad \chi_{\mathrm{Br}}=-27.80[17], \quad \chi_{\mathrm{COOH}}=-17.20[17], \\
& \chi_{\mathrm{NH}_{2}}=-10.94[14] \text { and } \chi_{\mathrm{OH}}=-6.70[14] .
\end{aligned}
$$

TABLE I

Observed and calculated susceptibilities (in $10^{-6} \mathrm{emu} \mathrm{mol}{ }^{-1}$ ).

\begin{tabular}{l|c|c|c}
\hline \hline \multicolumn{1}{c|}{ Compound } & $\chi_{\mathrm{M}}^{\text {obs }}$ & $\chi_{\mathrm{M}}^{\text {calc }}$ & $\Delta \chi_{\mathrm{M}}$ \\
\hline 3,5-dibromo- $p$-aminobenzoic acid & -129.03 & -130.94 & -1.91 \\
$\mathrm{Br}_{2} \mathrm{C}_{6} \mathrm{II}_{2}\left(p-\mathrm{NH}_{2}\right)$ COOII & & & \\
3,5 -dibromo- $p$-hyydroxybenzoic acid & -124.97 & -126.70 & -1.73 \\
$\mathrm{Br}_{2} \mathrm{C}_{6} \mathrm{H}_{2}(p$-OII $)$ COOII & & &
\end{tabular}

The compounds under investigation are strongly hydrogen bonded in their crystalline state. For the first compound, on one side, molecule $I$ is bonded to another molecule of type I by O-II. . O hydrogen bonds of length $2.63 \AA$ [15] and to molecules of type II by weak N-II. . O hydrogen bonds of length $3.07 \AA$, whereas 
on the other side it is bonded to molecules of type II by N-H. . O hydrogen bonds of length $2.90 \AA$. Molecule II is, however, held by strong O-H. . O hydrogen bonds of length $2.58 \AA$ and N-H. . O hydrogen bonds of length $2.90 \AA$ on one side and by weak N-HI. . O bonds of length $3.07 \AA$ on the other side, see Fig. 1. The crystal structure of the second compound is not determined.

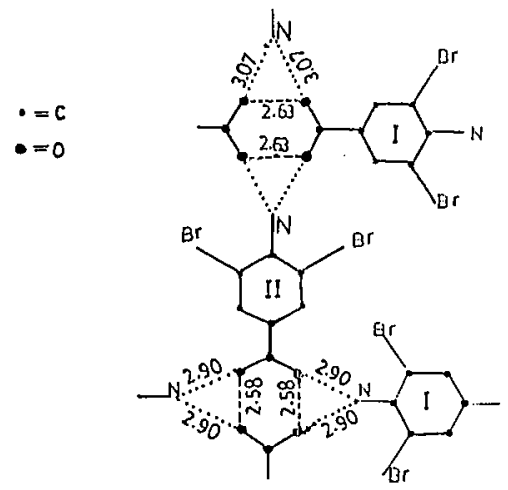

Fig. 1. The hydrogen bonding system in 3,5-dibromo-p-aminobenzoic acid (after A.K. Pant, Ref. [15]).

It is of interest to correlate the magnetic data of the two compounds with the hydrogen bonding system. One can investigate the effect of the hydrogen bonding on the magnetic properties of the molecule by comparing the delocalization anisotropy of the molecule $\left(\Delta K^{\prime}\right)$ with that of the benzene ring. According to Mason [12] and Craig [19] the hydrogen bond can give rise to electron delocalization resulting in magnetic anisotropy. The delocalization anisotropy $\Delta K^{\prime}$ is given by

$$
\Delta K^{\prime}=K^{\perp}-\sum \chi_{\text {atomic }} .
$$

The values of the atomic susceptibilities used for the calculation of $\sum \chi_{\text {atomic }}$,are: $\chi_{\mathrm{C}}=-7.40$ [12], $\chi_{\mathrm{H}}=-2.00$ [12], $\chi_{\mathrm{O}}=-4.60$ [20], $\chi_{\mathrm{Br}}=-27.80$ [17], and $\chi_{\mathrm{N}}=-7.30[14]$. On the other hand, the average susceptibilities used for the calculations of $K^{\|}$, Table II, are

$$
\begin{array}{llrl}
\chi_{\mathrm{C}}^{\|}(\mathrm{Av}) & =-3.23[6], & & \chi_{\mathrm{H}}^{\|}(\mathrm{Av})=-2.69[6], \\
\chi_{\mathrm{O}}^{\|}(\mathrm{Av})=-3.54[21], & & \chi_{\mathrm{O}}^{\|}(\mathrm{Av})=-4.50[12,19], \\
\chi_{\mathrm{N}}^{\|}(\mathrm{Av})=-4.00[12,19], & \chi_{\mathrm{Br}}^{\|}(\mathrm{Av})=-27.80[22] .
\end{array}
$$

For the benzene ring the value of $\Delta K^{\prime}$ has been considered both theoretically and experimentally. The theoretical value as calculated by Edward and McWeeny is -37.8 [5] which is in agreement with the value of -38.00 given by Craig [19]. The experimental value is -38.20 [23]. The method used in this paper gives the value of -39.06 . As one can see from the data shown in Table II, the value of $\Delta K^{\prime \prime}$ of any of the two compounds under investigation is numerically higher than that of the benzene ring. The additional delocalization and hence additional magnetic anisotropy is therefore due to the formation of the hydrogen bonding in these molecules. 
TABLE II

The principal molecular susceptibilities for $\mathrm{Br}_{2} \mathrm{C}_{6} \mathrm{H}_{2}\left(p-\mathrm{NII}_{2}\right) \mathrm{COOII}$ (I) and $\mathrm{Br}_{2} \mathrm{C}_{6} \mathrm{H}_{2}(p-\mathrm{OH}) \mathrm{COOII}$ (II) $\left(K^{-1}\right.$ and $\left.K^{\perp}\right)$ and the contribution of hydrogen bonding (in $10^{-6}$ emu $\mathrm{mol}^{-1}$ ).

\begin{tabular}{|c|c|c|c|c|c|c|c|c|}
\hline & $\begin{array}{c}K^{-1}= \\
\sum \chi_{A}+n \alpha\end{array}$ & $\begin{array}{c}K^{-1 \|}= \\
\sum x^{\|(A v)}\end{array}$ & $K^{\prime} \|$ (mean) & $K^{-1}$ & $\Delta K^{\prime}$ & $\Delta^{a}$ & $\Delta K_{\text {semi }}$ & $\Delta^{* b}$ \\
\hline I & -107.30 & --103.69 & -105.49 & --176.11 & -42.21 & -3.15 & --70.62 & -5.62 \\
\hline II & -102.60 & -100.54 & -101.57 & -171.77 & -42.57 & -3.51 & -70.20 & -5.20 \\
\hline
\end{tabular}

The principle of the second method used for studying the effect of the hydrogen bonding is to compare the semi-experimental magnetic anisotropy of the molecule $\left(\Delta K_{\text {semi }}\right)$ with the calculated one $\left(\Delta K_{\text {calc }}\right)$. This method is usually preferable because, according to Lashccn [24], the experimental crrors in $\chi_{\mathrm{M}}^{\mathrm{obs}}$ may rcsult in some errors in the absolute valucs of $K^{\perp}$ and $K^{l l}$, but even a $10 \%$ crror in $\chi_{M}^{\text {obs }}$ makes an almost negligible error in $\Delta K_{\text {semi }}$. Fortunately, the magnetic anisotropy of the benzene ring has been investigated both theoretically and experimentally. The calculated values are $-60.90[6],-60.24[25]$ and -60.00 [8], while the experimental value is $-59.70[8,23]$. In the present work the magnetic anisotropy is taken as -60.00 . For the COOH group, its magnctic anisotropy is -5.00 [26]. Thus the calculated value of the magnetic anisotropy of any of the two compounds is therefore -65.00 . From Table II one can see that the numerical increase in the magnetic anisotropy per one molecule is about 5 . Thus for two molecules, i.e. one dimer, the combined effect is about 10. This numerical increase in the magnetic anisotropy is altributed to the electrons flowing in the rings formed by the hydrogen bonding, i.e. the so-called ring currents.

For the first compound, the crystal structure as well as the coefficients of thermal expansion were determined [15]. Since the compound belongs to an orthorhombic system, therefore, the axes of the triaxial ellipsoid coincide with the crystal axes. Thus the direction cosines of the $K^{\prime}$ 's values, $\cos \alpha_{i}, \cos \beta_{i}$, and $\cos \gamma_{i}$, $i=1,2$, and 3 , are calculated from the atomic coordinates as given by the X-ray structure.

The squares of the direction cosines are

$$
\begin{aligned}
& \cos ^{2} \alpha_{i}=0.0000,0.9964,0.0036, \\
& \cos ^{2} \beta_{i}=0.7454,0.0004,0.2534, \\
& \cos ^{2} \gamma_{i}=0.2545,0.0026,0.7425, \quad i=1,2, \text { and } 3 .
\end{aligned}
$$

Assuming that $K_{1} \cong K_{2} \cong K^{\prime l}$ and using the set of equations [9] relating crystal susceptibilities for an orthorhombic system $\left(\chi_{a}, \chi_{b}\right.$, and $\left.\chi_{c}\right)$ with the principal molecular susceptibilities $\left(K^{\|}\right.$and $K^{\perp}$ ), we get the values (in $10^{-6} \mathrm{emu}$ $\mathrm{mol}^{-1}$ )

$$
\chi_{a}=-105.74, \chi_{b}=-123.31, \text { and } \chi_{c}=-157.83 .
$$


Thus for an orthorhombic system or for any system whose orientation of the triaxial ellipsoid representing a second rank tensor property is known, it is possible (for a very good degree of approximation) to calculate the crystal susceptibilities as described above.

\section{References}

[1] F. London, J. Phys. Rad. 8, 397 (1937).

[2] H. Akamatu, Y. Matsunaga, Bull. Chem. Soc. Jpn. 26, 364 (1953); 29, 800 (1956).

[3] J.A. Pople, Mol. Phys. 1, 175 (1958).

[4] H. IIaberditzal, Angew. Chem. Int. Edit. 5, 288 (1966).

[5] T.G. Edwards, R. McWeeny, Chem. Phys. Lett. 10, 283 (1971).

[6] A.T. Amos, F. Roberts, J. Chem. Phys. 50, 2375 (1969).

[7] P. Lazzeretti, F. Taddci, J. Chem. Soc. Faraday Trans. II 68, 1825 (1972).

[8] P.II. Blustin, Mol. Phys. 36, 297 (1978).

[9] M.M. Abdel-Kader, Chem. Phys. Lell. 93, 297 (1982); Acla Phys. Pol. A63, 539 (1983); Mol. Phys. 48, 1145 (1983).

[10] J.A.N.F. Gomes, Mol. Phys. 40, 765 (1980).

[11] J. Iloarau, Ann. Chim. 13, 544 (1956).

[12] R. Mason, Mol. Phys. 4, 191 (1961); Acta Crystallogr. 14, 720 (1961).

[13] E.R. Long, J.D. Memory, J. Chem. Phys. 65, 2918 (1976).

[14] M.M. Abdel-Kader, Bull. Chcm. Soc. Jpn. 63, 586 (1990).

[15] A.K. Pant, Acla Crystallogr. 19, 440 (1965).

[16] M.M. Abdel-Kader, Acla Phys. Pol. A65, 273 (1984).

[17] C.M. French, Trans. Faraday Soc. 50, 1320 (1954).

[18] S. Sriraman, R. Sabesan, Bull. Chem. Soc. Jpn. 36, 547 (1963).

[19] D.P. Craig, M.L. IIcfernan, N.L. Paddock, J. Chem. Soc., Part I , 1376 (1961).

[20] C.K. Ingold, Structure and Mechanism in Organic Chemistry, Cornel Univ. Press, New York 1953, p. 60.

[21] D.W. Davies, Nalure 190, 1102 (1961).

[22] M.M. Abdel-Kader, M.Sc. Thesis, Cairo Univ. 1976.

[23] R. Benarsi, P. Lazzeretli, F. Taddei, J. Phys. Chem. 79, 484 (1975).

[24] M.A. Lasheen, Philos. Trans. R. Soc. (London) 256, 357 (1964).

[25] J. IIoarau, N. Lumbroso, A. Pacault, Comp. Red. Acad. Sci. (Paris) 242, 1702 (1956).

[26] A. Mookerje, D. Neogy, Bull. Chem. Soc. Jpn. 34, 897 (1961). 